\title{
Predicting the impact of COVID-19 interruptions on transmission of gambiense human African trypanosomiasis in two health zones of the Democratic Republic of Congo
}

\author{
Maryam Aliee ${ }^{a, *, \dagger}$, Soledad Castaño ${ }^{b, \dagger}$, Christopher N. Davis $\mathbb{1 D}^{a}$, Swati Patel ${ }^{c}$, Erick Mwamba Miaka ${ }^{d}$, \\ Simon E. F. Spencer ${ }^{c}$, Matt J. Keeling ${ }^{\mathbb{a} a}$, Nakul Chitnis ${ }^{b}$, and Kat S. Rock (iD)
}

\author{
a Zeeman Institute (SBIDER), University of Warwick, Mathematical Sciences Building, Gibbet Hill Road, Coventry, CV4 7AL, UK; \\ ${ }^{b}$ Department of Epidemiology and Public Health, Swiss Tropical and Public Health Institute, Socinstrasse 57, Postfach, CH-4002 Basel, \\ Switzerland; 'Department of Statistics, University of Warwick, Mathematical Sciences Building, Gibbet Hill Road, Coventry, CV4 7AL, UK; \\ dProgramme National de lutte contre la THA (PNLTHA), Kinshasa 2, Democratic Republic of the Congo \\ *Corresponding author: Tel: 02476 573360; E-mail: Maryam.Aliee@warwick.ac.uk \\ †These authors contributed equally.
}

Received 20 October 2020; revised 18 January 2021; editorial decision 19 January 2021; accepted 19 January 2021

\begin{abstract}
Many control programmes against neglected tropical diseases have been interrupted due to the coronavirus disease 2019 (COVID-19) pandemic, including those that rely on active case finding. In this study we focus on gambiense human African trypanosomiasis (gHAT), where active screening was suspended in the Democratic Republic of Congo (DRC) due to the pandemic. We use two independent mathematical models to predict the impact of COVID-19 interruptions on transmission and reporting and achievement of the 2030 elimination of transmission (EOT) goal for gHAT in two moderate-risk regions of the DRC. We consider different interruption scenarios, including reduced passive surveillance in fixed health facilities, and whether this suspension lasts until the end of 2020 or 2021. Our models predict an increase in the number of new infections in the interruption period only if both active screening and passive surveillance were suspended, and with a slowed reduction-but no increase-if passive surveillance remains fully functional. In all scenarios, the EOT may be slightly pushed back if no mitigation, such as increased screening coverage, is put in place. However, we emphasise that the biggest challenge will remain in the higher-prevalence regions where EOT is already predicted to be behind schedule without interruptions unless interventions are bolstered.
\end{abstract}

Keywords: COVID-19, elimination of transmission, gambiense human African trypanosomiasis (gHAT), mitigation, modelling

\section{Introduction}

The threat posed by the coronavirus disease 2019 (COVID-19) pandemic is not limited to the direct consequences of the disease itself. In addition to the economic burden that many countries are facing due to lockdowns, the public health measures initiated to suppress transmission of severe acute respiratory syndrome coronavirus 2 (SARS-CoV-2) may have an additional impact on the ability to control other infectious diseases. A disruption in the usual activities of health services has the potential to lead to an increased loss of life as surveillance and access to diagnostics and treatment is more limited. ${ }^{1}$ These challenges to public health systems will affect Africa disproportionately, with COVID-19 providing an additional burden to many already fragile health systems. ${ }^{2}$ Previous modelling studies have suggested that COVID-19-related interruptions to malaria control could lead to substantially higher case and death numbers in Africa in the near future..$^{3,4}$

Gambiense human African trypanosomiasis (gHAT) is a vectorborne disease of Central and West Africa, which is typically fatal when left untreated, for which the delivery of interventions has already been impacted by the COVID-19 pandemic. A primary intervention to control gHAT is the use of active screening (AS), where at-risk populations in hard-to-reach locations are tested using serological diagnostics followed by subsequent case confirmation and treatment. ${ }^{5}$ In April 2020, the World Health Organization (WHO) recommended that active case-finding activities and mass treatment campaigns for neglected tropical diseases should be postponed until further notice. ${ }^{6}$ The lack of AS results in a reliance on the passive healthcare system and individuals self-presenting to health centres following the onset of symptoms. This will likely lead to diagnosis at a later stage of this

(c) The Author(s) 2021. Published by Oxford University Press on behalf of Royal Society of Tropical Medicine and Hygiene. This is an Open Access article distributed under the terms of the Creative Commons Attribution License (http://creativecommons.org/licenses/by/4.0/), which permits unrestricted reuse, distribution, and reproduction in any medium, provided the original work is properly cited. 
lethal disease, with additional uncertainty about whether the pandemic will also lead to patient fears about travelling to health centres and a reduced priority of the disease for diagnosis in health centres due to COVID-19 testing. ${ }^{7-9}$

For gHAT, interruptions in AS in the past have led to increased infections in subsequent years. The 2014-2015 Ebola outbreak in Guinea, which completely interrupted AS and resulted in 'partial' passive surveillance, coincided with a decrease in reported gHAT incidence that was likely due to poor surveillance rather than a true reduction in infection numbers. ${ }^{7}$ We can also learn from other interruptions of AS: a period of no active screening in 2007-2008 in Mandoul, Chad, also led to increased passive case detections in 2008, with modelling suggesting there was an increase in transmission. ${ }^{10} \mathrm{~A}$ challenge for controlling intensified disease management neglected tropical diseases (IDM NTDs) is the strong link between control activities (i.e. screening) and surveillance through case reporting, so a reduction in reported cases can be indicative of either success (if surveillance is strong) or reduced intervention efforts. ${ }^{11-13}$

There is a risk that the interruption of gHAT interventions will impact the goal of elimination of transmission (EOT) of gHAT by 2030 , which has been set by the WHO. ${ }^{14,15}$ While modelling suggests that it is already unlikely that all areas will achieve this goal, ${ }^{16,17}$ there is now the potential for further delays. To quantify the length of a delay in time to reach EOT caused by COVID-19 interruptions, and the corresponding increase in transmission and number of cases and possible deaths, we used two independent stochastic models for gHAT infection in two distinct transmission settings in the Democratic Republic of Congo (DRC), the country with the highest case burden. We also simulate mitigation scenarios by accelerating active screening after interruptions to study potential readjustments.

\section{Methods}

\section{Study populations}

In this work we focus on administrative regions in Kwilu province (within the former Bandundu province), which was the highest endemic province in the country in 2016. We selected two different health zones within Kwilu, Bagata and Mosango, both classified as moderate risk in 2016 (1-10 reported cases per 10000 people per year ${ }^{14}$ ), although Bagata has had higher case reporting (see SI-1 Figures 2,5, 7). Both health zones are similar in population size (estimated at 121433 for Mosango and 165990 for Bagata in 2015). ${ }^{18}$ The health zones have had regular active screening, with Bagata generally observing higher coverage. In Bagata the mean coverage during 2014-2018 was approximately $35 \%$ and the maximum was approximately $45 \%$; in Mosango the mean coverage during 2014-2018 was approximately $33 \%$ and the maximum was $60 \%$. We chose regions in which vector control has not yet been implemented in order to better study the effect of screening interruptions.

\section{Model overview}

The impact of interruptions of screening programs was studied using two independently developed stochastic models of gHAT infection, model S (Swiss Tropical and Public Health Institute) and model W (University of Warwick), developed independently and previously described in other modelling studies. ${ }^{19-23}$ Both these models take into account different stages of the disease and transmission between vectors and humans who might be at low or high risk of exposure to vector bites. They also allow for various screening programmes, including passive surveillance (PS) and AS, through which infected people may be identified and treated (more details, including a table with the similarities and differences in the models and simulations, can be found in SI-1 [see Table 8] $)^{20,24}$.

The parameters used in the stochastic models were taken from fitting the corresponding deterministic models to the human case screening data recorded for the years 2000-2016 in each health zone, except for the model $S$ calibration for Bagata, which used data for 2000-2018 (see SI-1 and SI-2) ${ }^{20}$. The fits assumed a $3 \%$ annual increase of the human population according to the available estimates and an increase in the passive detection rate over time-an improvement that is backed by both anecdotal evidence and previous modelling studies ([data aggregation] ${ }^{20,24}$ ). Annual rates of active screening were captured directly from the data for the period 2000-2018. The stochastic simulations use 200 sets of parameter values of the posterior distributions of specific health zones and each model generates 200000 stochastic realisations (1000 stochastic realisations for each of the 200 parameter sets). The model outputs compared with historic data can be seen in SI-1 (Figures 2 and 4).

\section{Interruption scenarios}

For a no-interruption baseline, we assume AS and PS interventions continue indefinitely from 2019 with the same number of people screened annually, given by the mean value of the last 5 y (2014-2018). We then consider six potential interruption scenarios of gHAT activities due to COVID-19. We assume all interruptions start at the beginning of April 2020, but they may last until either the end of 2020 or 2021 . The interruptions may disrupt either AS or both AS and PS. While AS is assumed to be fully suspended within the interruption period, PS may be partially operating, going back to the detection capacity before modelled improvement; for model $W$ this was the PS level in 1998 and for model $S$ this was for 2000. Table 1 summarises these six interruption scenarios. The interventions are reinstated to the baseline values (mean AS and full PS) after the interruption period. Moreover, we studied identical scenarios with mitigation, where AS is set to the maximum coverage observed in the data between 2000 and 2018, after interruption finishes. Simulating these 13 scenarios allows us to study possibilities of catching up to previously expected progress or even accelerating towards the 2030 goal.

\section{Results}

We compared infection dynamics of different scenarios by following the predicted annual number of new human infections, reported cases corresponding to both passive surveillance and active screening and the deaths caused by gHAT. Figures 1 and 2 show the mean dynamics over time, averaged for all realisations, of all 13 scenarios as described in Table 1 . In some cases the mean is substantially higher than the median and so we provide 
Table 1. gHAT strategies and interruption scenarios (due to COVID-19) considered in this simulation study

\begin{tabular}{|c|c|c|c|c|}
\hline Name & Interruption length & $\begin{array}{l}\text { AS during } \\
\text { interruption }\end{array}$ & PS during interruption & $\begin{array}{l}\text { Mitigation after } \\
\text { interruption }\end{array}$ \\
\hline Baseline & None & Mean of 2014-2018 & $\begin{array}{l}\text { Full (current detection rate } \\
\text { is fitted) }\end{array}$ & Not applicable \\
\hline $2020 \mathrm{~A}$ & $\begin{array}{l}\text { From April } 2020 \\
\text { until end of } 2020\end{array}$ & None & Full & $\begin{array}{l}\text { No, mean AS reinstated } \\
\text { Yes, maximum AS begins }\end{array}$ \\
\hline 2020 A+PP & $\begin{array}{l}\text { From April } 2020 \\
\text { until end of } 2020\end{array}$ & None & $\begin{array}{l}\text { Partial (levels in } 2000 \text { for } \\
\text { model S and } 1998 \text { for } \\
\text { model W) }\end{array}$ & $\begin{array}{l}\text { No, mean AS reinstated } \\
\text { Yes, maximum AS begins }\end{array}$ \\
\hline $2020 A+P$ & $\begin{array}{l}\text { From April } 2020 \\
\text { until end of } 2020\end{array}$ & None & None & $\begin{array}{l}\text { No, mean AS reinstated } \\
\text { Yes, maximum AS begins }\end{array}$ \\
\hline $2020-21 \mathrm{~A}$ & $\begin{array}{l}\text { From April } 2020 \\
\text { until end of } 2021\end{array}$ & None & Full & $\begin{array}{l}\text { No, mean AS reinstated } \\
\text { Yes, maximum AS begins }\end{array}$ \\
\hline 2020-21 A+PP & $\begin{array}{l}\text { From April } 2020 \\
\text { until end of } 2021\end{array}$ & None & Partial & $\begin{array}{l}\text { No, mean AS reinstated } \\
\text { Yes, maximum AS begins }\end{array}$ \\
\hline 2020-21 A+P & $\begin{array}{l}\text { From April } 2020 \\
\text { until end of } 2021\end{array}$ & None & None & $\begin{array}{l}\text { No, mean AS reinstated } \\
\text { Yes, maximum AS begins }\end{array}$ \\
\hline
\end{tabular}

baseline projections in the SI-1, showing the mean and median as well as the $95 \%$ prediction intervals for the two models and both health zones (see SI-1 Figures 3 and 6). These wider prediction intervals explain why the mean number of new transmissions in model S can be greater than in model W (Figure 2), even though there is a higher probability of achieving EOT for model $S$ at any time in Bagata and during 2020-2030 in Mosango (Figure 3).

Similar trends can be recognised for the two health zones of Bagata and Mosango despite their different prevalence and infection dynamics. The results from both models and both health zones predict a significant increase in the mean number of new infections following suspension of both AS and PS. On the other hand, the number of reported cases decreases during the interruption period but then resurges in the following years, especially in mitigated scenarios where active screening is reinstated at the maximum historic coverage. More importantly, our models predict higher death rates during and after the interruptions. If PS continues, either at full or reduced capacity, our simulations indicate that the number of infections is unlikely to increase during the interruption; however, progress towards elimination of transmission could slow or stagnate during interruption. Putting these results together, the loss is more pronounced for longer interruptions (until the end of 2021) and, as expected, the worst response is observed when both active and passive activities are fully ceased. These results suggest retaining a minimum level of PS plays a significant role in controlling transmission, even if planned AS cannot go ahead. In the worst scenario, if all activities are suspended for 2 y (2020-21 A+P), mitigations are not expected to help catch up with the baseline before 2030 .

In addition to examining the expected infection and reporting dynamics, we also estimate the EOT probability by calculating the cumulative fraction of simulations reaching EOT over time for these health zones. In case of no interruptions, our models predict a fairly high probability (82\% chance for model W and $79 \%$ for model S) to achieve EOT in Mosango by 2030. Suspension of AS alone is not predicted to disturb that substantially (see Figure 3). This probability is clearly decreased, yet still $>70 \%$, in the worstcase scenario when all activities are suspended for 2 y even without mitigation. The median elimination year is delayed for almost $2 \mathrm{y}$ in this scenario. Mitigated programs can facilitate elimination and may even result in higher probabilities of achieving the EOT goal compared with the baseline scenario without interruption in the case that some PS was retained. In contrast to Mosango, reaching the 2030 EOT goal does not seem likely with the current programs in Bagata (29\% and 56\% probability for models W and $\mathrm{S}$, respectively), even without any pause in gHAT-related activities. However, EOT by 2030 becomes more unlikely when severe interruptions are introduced. Our predictions for Bagata suggest the median elimination year to be between 2029 and 2035, which could be delayed to 2032-2037 in the worst-case scenario.

To visualise the predicted delays in elimination, we present violin plots representing the probability that EOT is predicted to occur during a specific year (probability density function of EOT) in our simulations (Figure 4), which clearly show how the distributions are shifted towards later years for severe scenarios.

While the qualitative trends and even 2030 EOT probabilities for the two health zones are similar between models, we note that there are quantitative differences. In particular, model S has wider prediction intervals than model W (see SI-1 in Figures 2 and 4) and this is reflected in both the higher means for new transmission in both health zones, even though medians are lower, and in the wider violin plot distributions. 

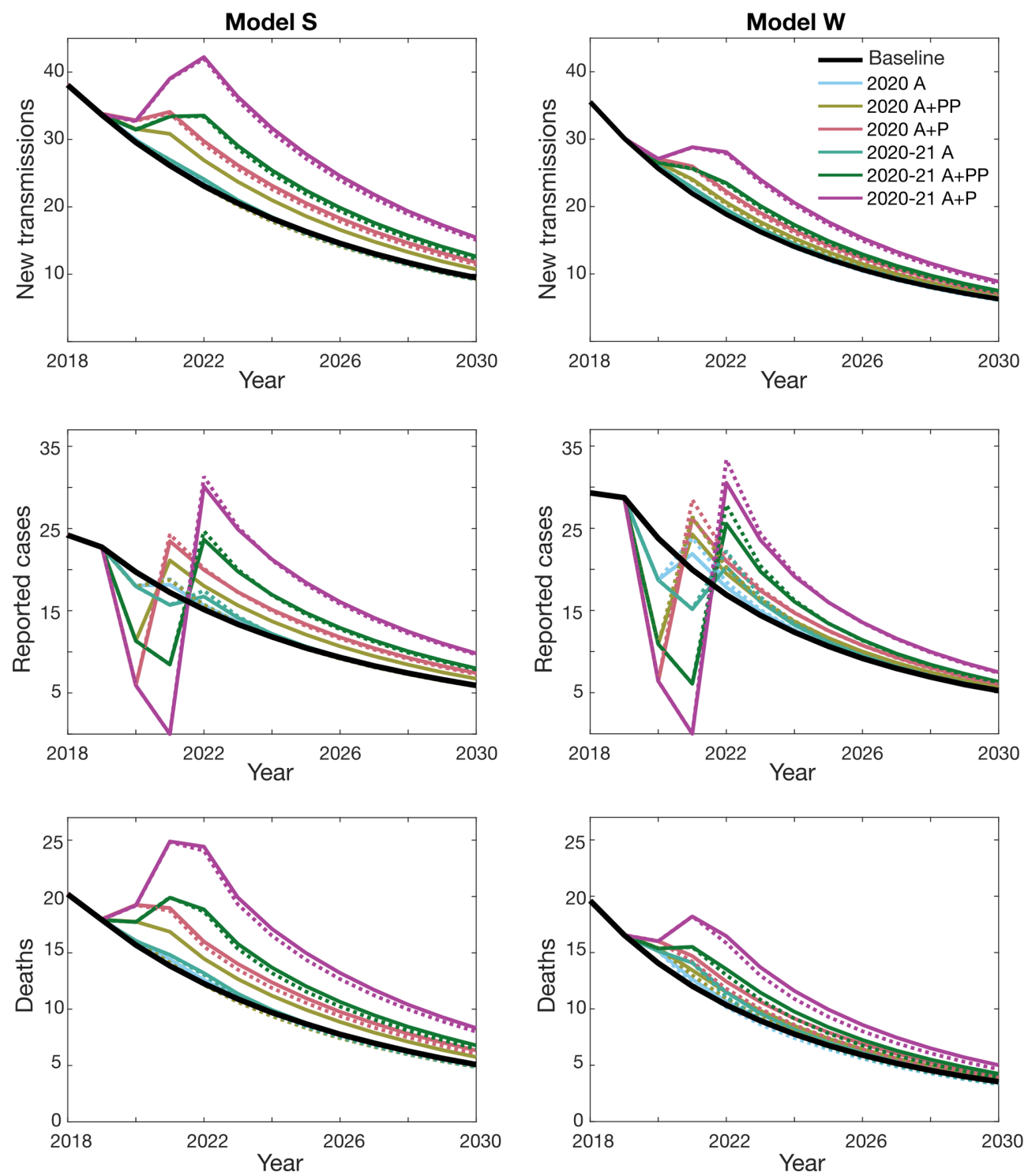

Figure 1. Predicted gHAT infections in the Bagata health zone based on different COVID-19 interruption scenarios. The graphs show the expected number of new transmissions, reported cases and number of deaths caused by the disease (mean values) for model $\mathrm{S}$ (left-hand side) and model W (right-hand side). The health zone population was 165990 in 2015. The baseline is shown as a black solid line. Individual interruption scenarios without mitigation are depicted by solid lines of different colours as indicated in the first panel. The corresponding mitigation scenarios are shown with dashed lines using comparable colours.

\section{Discussion}

Our analysis of two independent models highlights the possible damage caused by the suspension of gHAT interventions due to COVID-19. In the most severe scenario, we assume both AS and PS would be completely stopped until the end of 2021, which is predicted to delay EOT by an average of 2-3 y. However, the COVID-19 pandemic continues to evolve and it remains unclear when public health measures will be relaxed (or reinstated). In case of longer interruptions (not simulated here), we might expect more serious damage to the gains made to date by the elimination efforts.
The predictions made are specific to two health zones of the DRC-Mosango and Bagata; other health zones could behave differently, especially if current controls are playing a stronger role in reducing transmission. Our analysis predicts EOT may still be achieved in Mosango by 2030 thanks to the recent boost in AS coverage. Our predictions are more uncertain in Bagata. Model S suggests the 2030 EOT goal, which may have been achievable without any interruption, could be missed due to the interruptions if PS is reduced, however, model $\mathrm{W}$ predicts the elimination goal is unlikely to be met without intensifying interventions, even without COVID-19-related interruptions. The heterogeneous nature of active screening in gHAT-endemic 

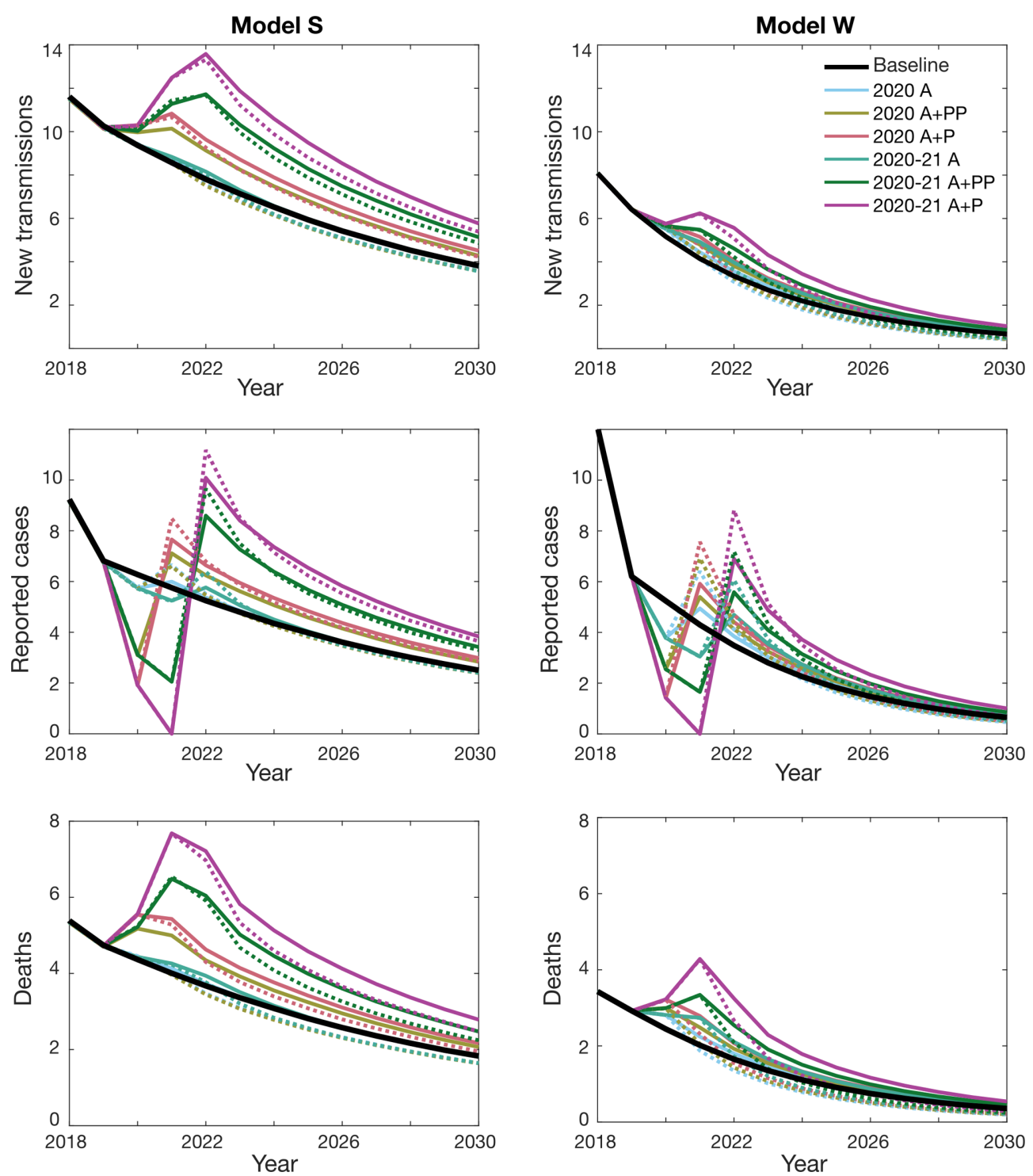

Figure 2. Predicted gHAT infections in the Mosango health zone based on different COVID-19 interruption scenarios. The graphs show the expected number of new transmissions, reported cases and number of deaths caused by the disease (mean values) for model $S$ (left-hand side) and model W (right-hand side). The health zone population was 121433 in 2015. The baseline is shown as a black solid line. Individual interruption scenarios without mitigation are depicted by solid lines of different colours as indicated in the first panel. The corresponding mitigation scenarios are shown with dashed lines using comparable colours.

areas and the underlying focal nature of disease transmission mean that results will be region specific for the delay in EOT and the necessity of mitigation strategies should be evaluated on this basis. ${ }^{14}$ Additionally, in Mosango and Bagata there has been no wide-scale vector control, and we would expect qualitatively different impacts in areas where this has already been implemented. In the Ebola outbreak of 2014-2015, the interruption to active screening and passive surveillance is thought to have caused an increase in transmission in the affected areas, except in places where small insecticide impregnated targets could be maintained, indicating tsetse control is likely protective during medical interruptions if it remains in situ. ${ }^{7,25}$
Moreover, our results suggest that retaining functioning passive surveillance, even partially, can help to avoid significant delays in EOT and prevent substantial increases in mortality. Even with a functional health system, it is unclear how pandemicinduced changes in health-seeking behaviour, in addition to redirecting limited health resources, will impact levels of passive case finding. Mitigation through increasing coverage of AS following interruption could also increase the probability of meeting the 2030 EOT goal. On the whole, our results suggest a milder impact of COVID-19-related delays on gHAT incidence and mortality than that suggested by similar studies on malaria. $^{3,4}$ 

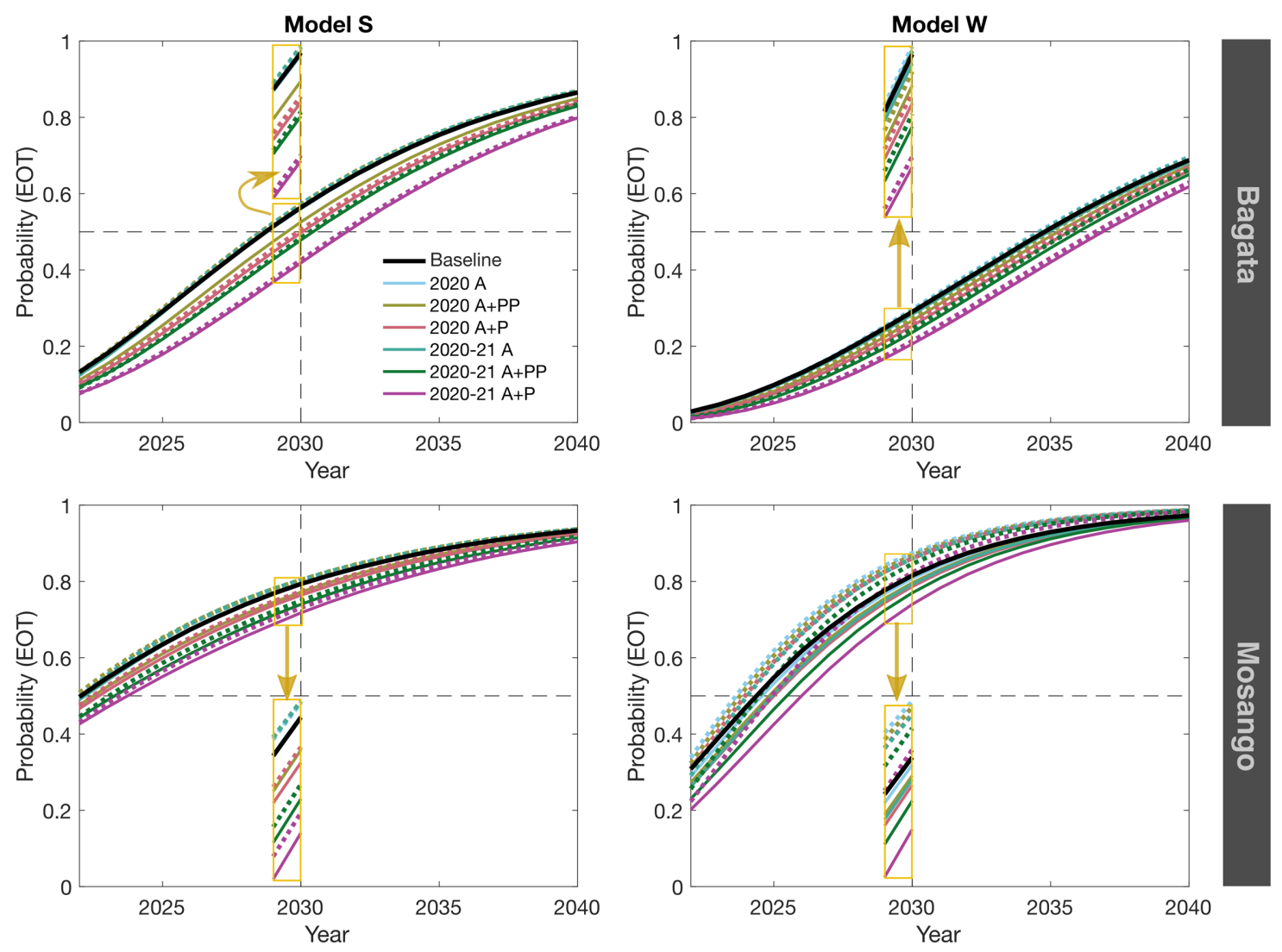

Figure 3. Cumulative probability of elimination of transmission by year. Predictions of both models showing the probability of achieving EOT by a given year are plotted for the period of 2022-2040 for the Bagata and Mosango health zones under different interruptions and the corresponding mitigation scenarios (maximum AS after the interruption) are shown by dashed lines. A zoomed version around 2030 is shown as an inset (yellow boxes).

The use of stochastic events in both presented model formulations enable the direct computation of EOT prediction within health zones by calculating the probability from multiple realisations of the infection process. However, we note that neither model considers the impact of an asymptomatic reservoir of human infection that is able to self-cure or how an animal reservoir may be able to sustain transmission, as the extent of these factors remains unknown. ${ }^{26,27}$ Although animal reservoirs and asymptomatic infections may have a substantial impact on the effectiveness of particular strategies in achieving EOT, they are unlikely to change the impact of COVID-19-related delays on achieving EOT. Furthermore, local EOTs for health zones will somewhat depend on EOTs in neighbouring regions, especially if there is substantial movement of people between locations. The modelling work here assumed that no importation of infection occurs, with health zones considered 'epidemiological islands'. Previous modelling work indicates there may be only small rates of importation between villages in endemic regions within the Kwilu province, ${ }^{21}$ but nearby regions of continued transmission could pose a threat of reintroduction into health zones that successfully achieve EOT.

The use of two different models has enabled us to account for some structural and parameter uncertainty due to the way the models were constructed and fitted to observed data. A brief summary of the model differences is given in SI-1
(Table 5). Through fitting to data, both models estimated the passive detection rates in 2000 and 2016 (see SI-1 Tables 1 and 5), with both models achieving comparable values for 2016 in both health zones. There was a noticeable difference in inferred base passive detection values for Bagata between the two models, leading to model S estimating an approximately 2.6-fold and 24-fold improvement between 2000 and 2016 for stages 1 and 2 , respectively, while model $W$ suggested that these improvements were more modest at around 4 times for stage 1 and 1.1 times for stage 2. These structural and parameter differences illustrate why it is unsurprising that the two models provide different projections for the baseline and interruption scenarios. Despite this, they do reach consensus that the interruptions considered here would be unlikely to represent a large setback for the programme. Furthermore, they provide similar quantitative estimates for the probability that each health zone will meet the EOT target by 2030 under baseline and suggest that Bagata in particular will need to have an intensified strategy to meet the EOT and achieve the goal. Neither model explicitly incorporates the use of the new oral drug fexinidazole; however, we believe that these results would be quite similar if compliance with the fexinidazole drug regime and parasite clearance were comparable to existing treatments (see SI).

These results provide a rather optimistic perspective of COVID19-related interruption on gHAT control. We stress that the 


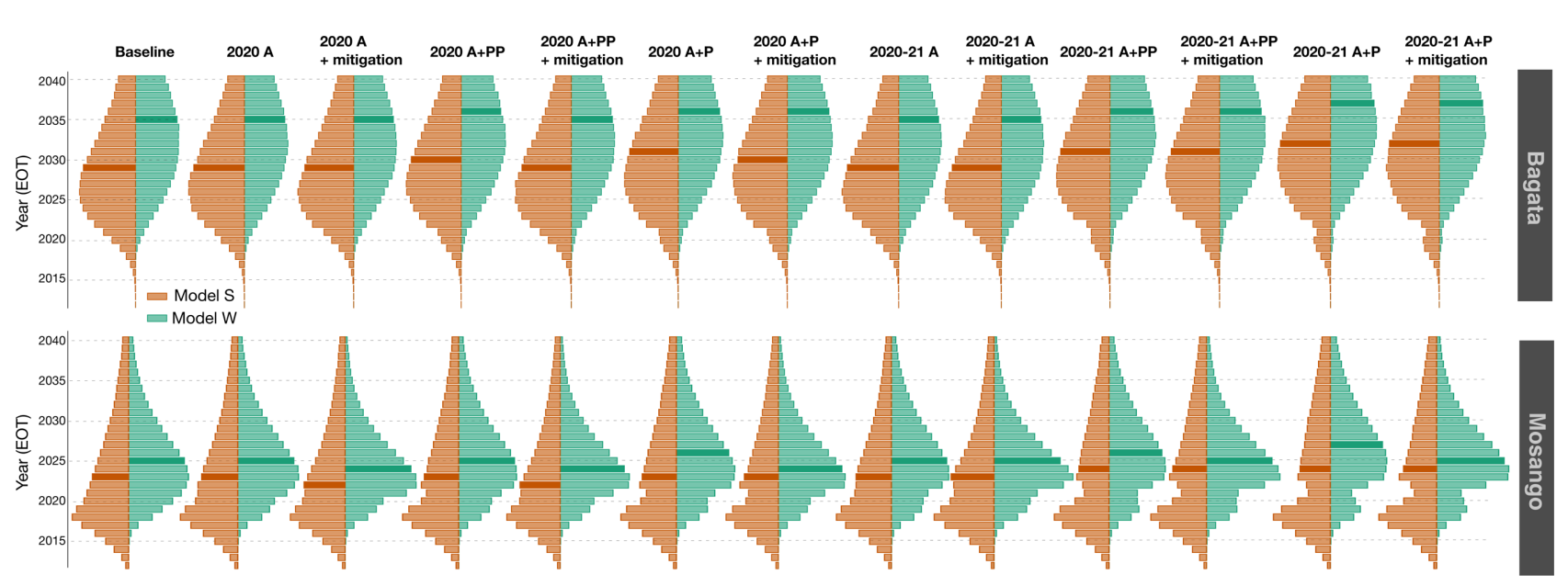

Figure 4. Distribution of the year in which EOT is predicted under different interruption scenarios. The violin plots compare the probability of EOT being achieved during each year and show model W (green) and model S (orange). Larger bars have higher probabilities of EOT being achieved in that year and the median elimination year is shown by a darker colour. The first and second rows correspond to Bagata and Mosango respectively.

cumulative impact of simultaneous programme interruptions for multiple diseases (NTDs and other infections) afflicting the same vulnerable populations could become an additional global health issue that deserves early attention and sustained control efforts.

\section{Supplementary data}

Supplementary data are available at Transactions online.

Authors' contributions: M.A. was responsible for study implementation, analysis and interpretation of data and writing the manuscript. SC was responsible for study implementation and analysis and interpretation of data. CND was responsible for analysis and interpretation of data and writing the manuscript. SP was responsible for analysis and interpretation of data. EMM was responsible for study design. SEFS and MJK were responsible for analysis and interpretation of data. NC was responsible for study design and analysis and interpretation of data. KSR was responsible for study design, analysis and interpretation of data and writing the manuscript. All authors read and approved the final manuscript.

Acknowledgements: The authors thank the PNLTHA for original data collection, the WHO for data access (in the framework of the WHO HAT Atlas). Calculations of model S were performed at the sciCORE Center for Scientific Computing at the University of Basel (http://scicore.unibas.ch/). We thank Ron Crump and Ching-I Huang for original model W fitting.

Funding: This work was supported by the Bill and Melinda Gates Foundation through the NTD Modelling Consortium (OPP1184344) and through the Human African Trypanosomiasis Modelling and Economic Predictions for Policy (HAT MEPP) project (OPP1177824). The funders had no role in the study design, data collection and analysis, decision to publish or preparation of the manuscript.

Competing interests: None declared.

Ethical approval: Not required.
Data availability: We stated what data we used, include the necessary ones and cited the references properly in the main text and the SI.

\section{References}

1 Wang J, Xu C, Wong YK, et al. Preparedness is essential for malaria-endemic regions during the COVID-19 pandemic. Lancet. 2020;395(10230):1094-6.

2 Makoni M. Africa prepares for coronavirus. Lancet. 2020;395 (10223):483.

3 Hogan AB, Jewell BL, Sherrard-Smith E, et al. Potential impact of the COVID-19 pandemic on HIV, tuberculosis, and malaria in low-income and middle-income countries: a modelling study. Lancet Glob Health. 2020;8(9):e1132-41.

4 Sherrard-Smith E, Hogan AB, Hamlet A, et al. The potential public health consequences of COVID-19 on malaria in Africa. Nat Med. 2020;26(9):1411-6.

5 World Health Organization. Control and surveillance of human African trypanosomiasis. WHO TRS 984. Geneva: World Health Organization; 2013.

6 World Health Organization. Global Health Observatory. Available from: https://www.who.int/data/gho.

7 Camara M, Ouattara E, Duvignaud A, et al. Impact of the Ebola outbreak on Trypanosoma brucei gambiense infection medical activities in coastal Guinea, 2014-2015: a retrospective analysis from the Guinean national Human African Trypanosomiasis control program. PLoS Negl Trop Dis. 2017;11:e0006060.

8 Chanda-Kapata P, Kapata N, Zumla A. COVID-19 and malaria: a symptom screening challenge for malaria endemic countries. Int J Infect Dis. 2020;94:151-3.

9 Mitashi P, Hasker E, Mbo F, et al. Integration of diagnosis and treatment of sleeping sickness in primary healthcare facilities in the Democratic Republic of the Congo. Trop Med Int Health. 2015;20(1): 98-105.

10 Mahamat MH, Peka M, Rayaisse JB, et al. Adding tsetse control to medical activities contributes to decreasing transmission of sleeping 
sickness in the Mandoul focus (Chad). PLoS Negl Trop Dis. 2017;11(7):e0005792.

11 Coffeng LE, Le Rutte EA, Muñoz J, et al. Impact of changes in detection effort on control of visceral leishmaniasis in the Indian subcontinent. J Infect Dis. 2020;221(Suppl 5):S546-53.

12 NTD Modelling Consortium. The potential impact of programmes interruptions due to COVID-19 on 7 neglected tropical diseases: a modelling-based analysis. Gates Open Res. 2020;4:115. Available from: https://doi.org/10.21955/gatesopenres.1116665.1.

13 Toor J, Adams ER, Aliee M, et al. Predicted impact of COVID-19 on neglected tropical disease programs and the opportunity for innovation. Clin Infect Dis. 2020; doi: 10.1093/cid/ciaa933.

14 Franco JR, Cecchi G, Priotto G, et al. Monitoring the elimination of human African trypanosomiasis at continental and country level: update to 2018. PLoS Negl Trop Dis. 2020;14(5):e0008261.

15 Simarro PP, Cecchi G, Franco JR, et al. Monitoring the progress towards the elimination of gambiense human African trypanosomiasis. PLoS Negl Trop Dis. 2015;9(6):e0003785.

16 Huang CI, Crump RE, Brown P, et al. Shrinking the gHAT map: identifying target regions for enhanced control of gambiense human African trypanosomiasis in the Democratic Republic of Congo. medRxiv. 2020; https://doi.org/10.1101/2020.07.03.20145847.

17 NTD Modelling Consortium Discussion Group on Gambiense Human African Trypanosomiasis. Insights from quantitative and mathematical modelling on the proposed 2030 goal for gambiense human African trypanosomiasis (gHAT). Gates Open Res. 2020;3:1553.

18 Office for the Coordination of Humanitarian Affairs. Journees Nationales de Vaccination (JNV) Activities de vaccination supplementaire, RDC. https://data.humdata.org/about/license/legacy_ hrinfo [accessed May 2016].
19 Castaño MS, Aliee M, Mwamba Miaka E, et al. Screening strategies for a sustainable endpoint for gambiense sleeping sickness. J Infect Dis. 2019;221(Suppl 5):S539-45.

20 Crump RE, Huang CI, Knock E, et al. Quantifying epidemiological drivers of gambiense human African trypanosomiasis across the Democratic Republic of Congo. medRxiv. 2020; https://doi.org/10. 1101/2020.06.23.20138065.

21 Davis CN, Rock KS, Miaka EM, et al. Village-scale persistence and elimination of gambiense human African trypanosomiasis. PLoS Negl Trop Dis. 2019;13(10):e0007838.

22 Rock KS, Torr SJ, Lumbala C, et al. Quantitative evaluation of the strategy to eliminate human African trypanosomiasis in the Democratic Republic of Congo. Parasit Vectors. 2015;8:532.

23 Stone CM, Chitnis N. Implications of heterogeneous biting exposure and animal hosts on Trypanosomiasis brucei gambiense transmission and control. PLoS Comput Biol. 2015;11(10): e1004514.

24 Castaño MS, Ndeffo-Mbah ML, Rock KS, et al. Assessing the impact of aggregating disease stage data in model predictions of human African trypanosomiasis transmission and control activities in Bandundu province (DRC). PLoS Negl Trop Dis. 2020;14(1):e0007976.

25 Kagabadouno M, Camara O, Camara M, et al. Ebola outbreak brings to light an unforeseen impact of tsetse control on sleeping sickness transmission in Guinea. bioRxiv. 2018. DOI: 10.1101/202762.

26 Capewell P, Cren-Travaille C, Marchesi F, et al. The skin is a significant but overlooked anatomical reservoir for vector-borne African trypanosomes. Elife. 2016. https://doi.org/10.7554/eLife.17716.

27 Büscher P, Bart JM, Boelaert $M$, et al. Do cryptic reservoirs threaten gambiense-sleeping sickness elimination? Trends Parasitol. 2018;34(3):197-207. 